\title{
Sintilimab plus chemotherapy achieved symptom relief in stage IV lung squamous cell carcinoma with superior vena cava syndrome: a case report
}

\author{
Liuning Guo, Wendong Qu, Yating Wei, Lubiao Liang, Yang Tang, Cheng Chen", Xixian Ke ${ }^{\#}$ \\ Department of Thoracic Surgery, The Affiliated Hospital of Zunyi Medical University, Zunyi, China \\ \#These authors contributed equally to this work. \\ Correspondence to: Cheng Chen; Xixian Ke. Department of Thoracic Surgery, The Affiliated Hospital of Zunyi Medical University, 149 Dalian Road, \\ Zunyi 563000, China. Email: 29217036@qq.com; kexixian@zmu.edu.cn.
}

\begin{abstract}
Lung cancer is the second most common and mortality disease in the world. Most patients with lung cancer are already at the advanced stage when diagnosed. There are multiple treatments for advanced lung cancer. Among them, immunotherapy plus chemotherapy has gradually become the first-line treatment. Lung cancer has multiple complications, superior vena cava syndrome (SVCS) is a common complication of patients with advanced lung cancer. The current treatments include radiotherapy, chemotherapy, and stent implantation. Stent implantation has the disadvantages of invasiveness and poor efficacy. Immunotherapy, as an emerging treatment for tumors, has shown significant advantages in treating patients with advanced lung cancer. However, the treatment of advanced lung cancer with SVCS using immunotherapy has rarely been reported. Here, we reported a 48-year-old male patient with stage IV (T4N3M1) lung squamous cell carcinoma. After receiving 1 cycle (3 weeks) of comprehensive treatment with sintilimab plus chemotherapy, the tumor mass shrank by $30 \%$ to achieve partial response (PR), collateral circulation was formed, and most of the symptoms caused by SVCS disappeared. After 3 cycles of treatment, the tumor shrank by nearly $90 \%$, and the superior and inferior vena cava opened larger than before. Our case provides a novel treatment strategy for such patients.
\end{abstract}

Keywords: Lung squamous cell carcinoma; immunotherapy; sintilimab; superior vena cava syndrome (SVCS); case report

Submitted Nov 18, 2021. Accepted for publication Jan 14, 2022.

doi: 10.21037/apm-21-3674

View this article at: https://dx.doi.org/10.21037/apm-21-3674

\section{Introduction}

The mortality of lung cancer ranks first among all malignant tumors (1). Significant symptoms tend to occur in advanced lung cancer, and the prognosis of advanced lung cancer patients is poor. According to the eighth edition of TNM staging, the 1-year survival rate of patients with stage IVA lung cancer was only $23 \%$. About $2-4 \%$ of lung cancer patients have superior vena cava syndrome (SVCS) (2). SVCS is caused by the compression of the superior vena cava and manifests as neck swelling, dyspnea, trunk and/or upper limb swelling, facial swelling, chest pain, cough, thoracic venous side branch expansion, weight loss, jugular vein dilation, phrenic nerve palsy, and some emergency patients experience hoarseness, headache, confusion, dizziness, night sweats, hypoxia, hyponatremia, and syncope.

At present, the most common treatments for patients with stage IV lung cancer are radiotherapy, chemotherapy, and immunotherapy. Immunotherapy combined with chemotherapy is one of the most promising methods for the treatment of advanced lung cancer. The treatment of lung cancer accompanied by SVCS mainly includes radiotherapy, chemotherapy, and stent implantation. Although these treatments can improve patients' symptoms, there are also various limitations, such as the risk of stent displacement 

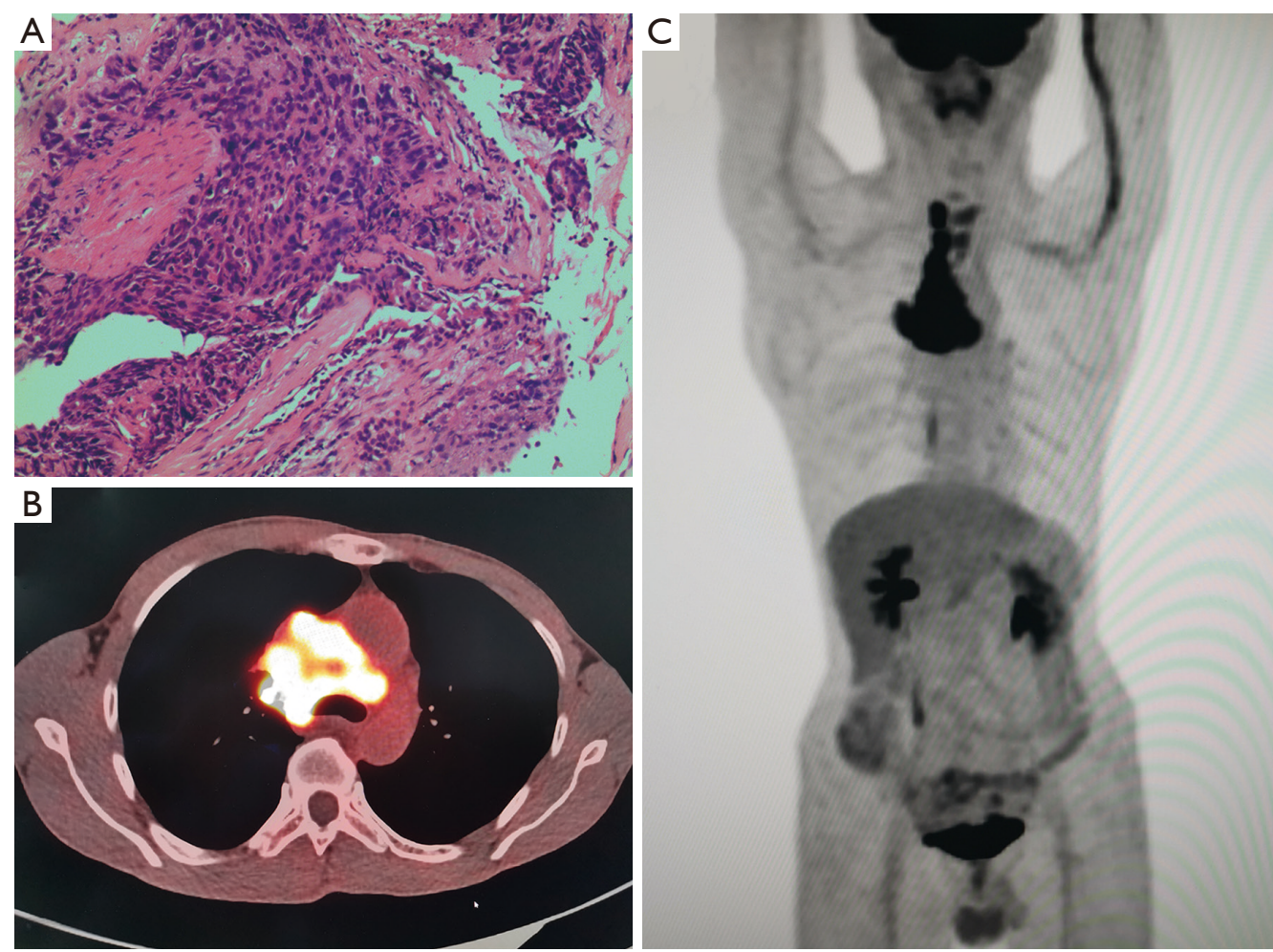

Figure 1 Pathology and PET/CT images of the patient. (A) HE staining of the primary lesion in the apical segment of the right upper lobe ( $\times 100)$; (B) PET/CT showed that the longitudinal and circular lymph nodes increased, enlarged, and merged into clusters with abnormally increased metabolism; (C) ${ }^{18} \mathrm{~F}$-deoxyglucose PET showed that the tumor was in a hypermetabolic state. PET, positron emission tomography; CT, computed tomography; HE, hematoxylin and eosin.

and occlusion after stent implantation and increased risk of superior vena cava rupture after radiotherapy (3).

Immunotherapy has opened a new chapter in the treatment of lung cancer. However, there are still few reports on the use of immunotherapy in lung cancer patients with comorbidities. In this case, sintilimab (PD-1 inhibitor) combined with platinum plus paclitaxel was administered to a patient with advanced lung squamous cell carcinoma. An encouraging therapeutic effect was achieved, with rapid shrinkage of the tumor mass, disappearance of SVCS, and clearance of the superior vena cava. As of the time of writing, there were no serious adverse reactions, warranting this case report. We present the following article in accordance with the CARE reporting checklist (available at https://apm.amegroups.com/article/view/10.21037/apm$21-3674 / \mathrm{rc})$.

\section{Case presentation}

In November 2020, a 48-year-old male was admitted to hospital with right neck swelling and facial edema for more than 20 days. At the time of admission, a mass in the right neck was found, which was about $5 \times 5 \mathrm{~cm}$ in size. The mass was tough, not tender, and had slightly poor moveability. The supraclavicular lymph nodes were not palpable and enlarged. The patient had facial edema and facial flushing symptoms but without chest pain, hemoptysis, hoarseness, night sweats, or significant weight loss, and no obvious medical or family history. The patient smoked (smoking index 400) but had no history of drinking. There was no diagnosis and treatment related to any cancer before hospitalization.

The positron emission tomography/computed tomography (PET/CT) images showed that there was an irregular reduced density area near the hilar of the right upper lobe, and the maximum value of standardized uptake value (SUV) was 7.8. There were multiple mediastinal lymph nodes which were partially fused, accompanied by abnormal fluorodeoxyglucose (FDG) uptake, and the maximum SUV was 14.2 (Figure 1). CT indicated that the tumor was at the apical segment of the right upper lobe, with mediastinal 

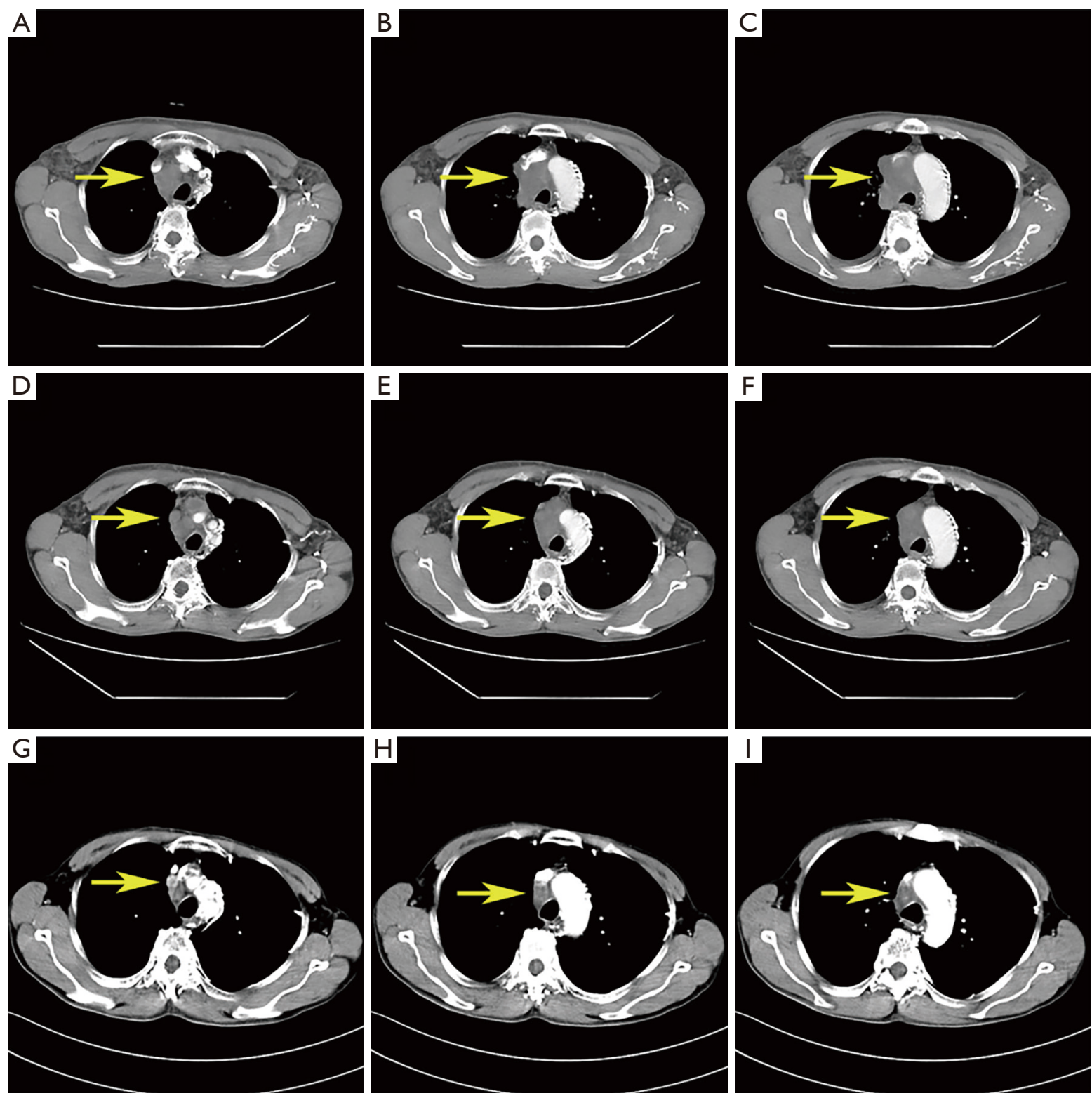

Figure 2 Enhanced CT of the chest showed changes in the mediastinal mass. (A-C) Enhanced CT images of the chest before neoadjuvant treatment; (D-F) enhanced CT images of the chest after 1 course of treatment showing that the tumor shrank; (G-I) enhanced CT images of the chest after 3 courses of treatment showing that the tumor size had significantly reduced and the superior vena cava was opened. The arrow indicates that the superior vena cava and bilateral innominate veins were invaded, and bilateral innominate vein tumors were detected. CT, computed tomography.

lymph node metastasis, and had invasion of superior vena cava and bilateral innominate veins. The pathological diagnosis with bronchoscopy was squamous cell carcinoma (Figure 1). The results of immunohistochemistry (the apical segment of the right upper lobe) also suggested squamous cell carcinoma, with the tumor cells showing the following characteristics: CK(+); TTF-1(-); Napsin A(-); P40(+); Syn(-); CD56(-); Ki67(hot spot 80\%+). The blood test found no obvious abnormalities. The other examinations suggested that the lung cancer invaded the blood vessels and the patient had mediastinal and cervical lymph node metastasis. According to the eighth edition of TNM staging, the patient 


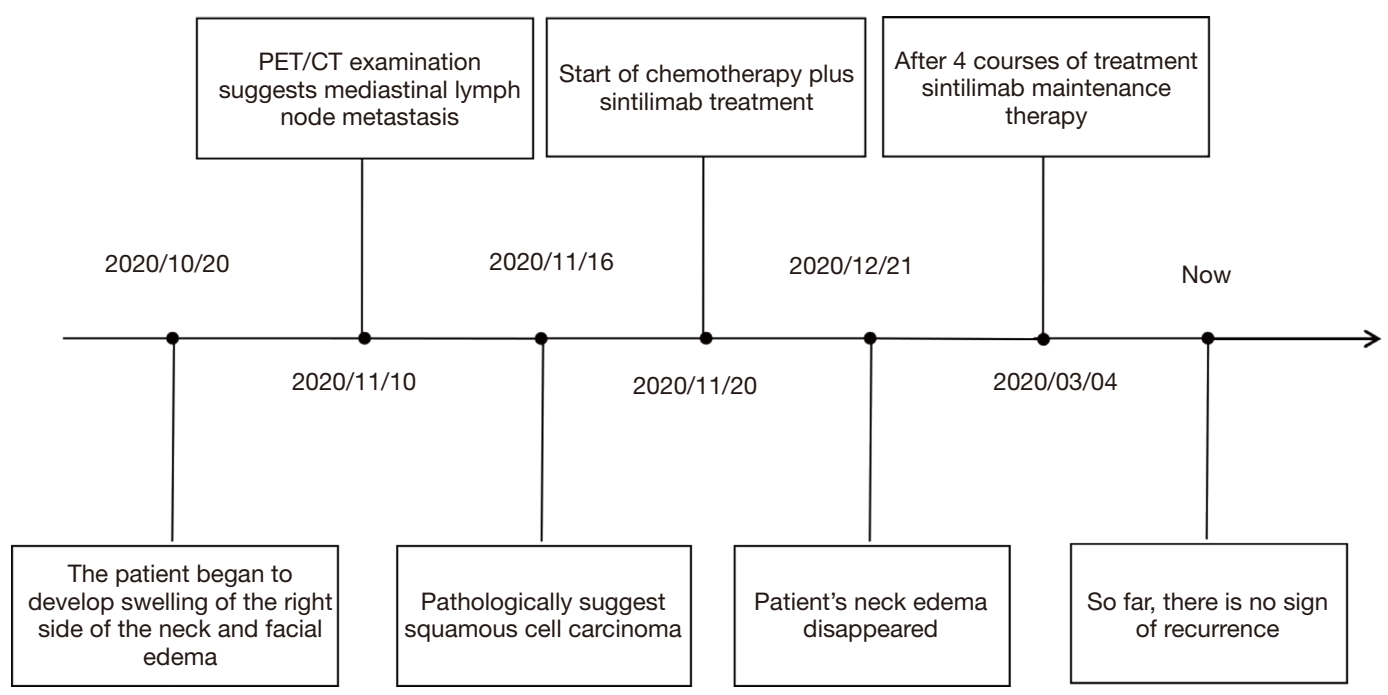

Figure 3 Timeline of diagnosis, treatment, and prognosis in this case. PET, positron emission tomography; CT, computed tomography.

was diagnosed with right upper lung T4N3M1 (stage IV) squamous cell carcinoma with SVCS.

After extensive discussions with the patient and his family about the potential risks of standard and non-standard treatment strategies, a combination of immunotherapy and chemotherapy was adopted. The patient received an intravenous infusion of carboplatin $400 \mathrm{mg}$, sintilimab $200 \mathrm{mg}$, and paclitaxel $180 \mathrm{mg}$ on $2020-12-15$, and was treated every 3 weeks for 4 courses. The adverse reactions, such as grade 2 diarrhea and nausea, were tolerable and well controlled, while no grade 3 or above adverse reactions occurred. Chest CT was performed to evaluate the treatment effect, which confirmed that the tumor was smaller than before, the SVCS symptoms were relieved, and the neck swelling disappeared after 1 course of treatment. The superior vena cava was unobstructed and recanalization (Figure 2). According to the Response Evaluation Criteria in Solid Tumors (RECIST) assessment criteria 1.1, it was judged as a partial response (PR).

At the time of writing, the patient was still undergoing regular immune monotherapy with sintilimab (Figure 3). The effectiveness of the treatment strategy still needs to be confirmed by long-term follow-up.

All procedures performed in this study were in accordance with the ethical standards of the institutional and/or national research committee(s) and with the Helsinki Declaration (as revised in 2013). Written informed consent was obtained from the patient for publication of this case report and accompanying images. A copy of the written consent is available for review by the editorial office of this journal.

\section{Discussion}

Lung squamous cell carcinoma is a common type of nonsmall cell lung cancer (NSCLC), accounting for $30 \%$ of lung cancer (4). The common metastases of advanced lung cancer include lymph node metastasis, brain metastasis, bone metastasis and liver metastasis. When the patient's mediastinal lymph nodes metastasize and compress or invade the superior vena cava, symptoms of superior vena cava will appear. At present, about $2-4 \%$ of lung cancer patients also have superior vena cava embolism. The treatment of these patients, including stent implantation, radiotherapy, chemotherapy, surgery, or other treatments, is based on the patient's condition. In the past, radiotherapy was considered to be the first-line treatment of SVCS. However, studies found that $20 \%$ of patients did not achieve symptom relief after radiotherapy, 5-30\% of which had symptom recurrence (5). Despite its poor efficacy, stent placement, which had the advantage of quickly relieving symptoms, is currently considered to be the first-line treatment (6). It was reported that $90 \%$ of patients who underwent stent placement show evident clinical improvement in 24 to 48 hours (7). However, stent placement also has complications such as stent displacement, obstruction, and pericardial tamponade. Furthermore, the surgery is invasive and not suitable for patients with a long life expectancy. Chemotherapy is non-invasive and is the first-line treatment for chemosensitive malignant tumors (6). Chemotherapy has the disadvantages of adverse reactions and needing time to relieve symptoms. In this 
case, SVCS was caused by squamous cell carcinoma. The tumor compressed the superior vena cava. Considering that the mild symptoms were tolerable, we chose chemotherapy combined with immune checkpoint inhibitors, aiming to rapidly shrink the patient's tumor so as to reduce the cost of treatment. After 1 course of treatment, the tumor mass shrank, collateral circulation formed, most of the symptoms disappeared, and clinical relief was achieved. The patient was assessed as PR according to the RECIST criteria.

For advanced squamous cell carcinoma, pembrolizumab combined with platinum-based chemotherapy is recommended as the first-line treatment. In the treatment of advanced NSCLC, sintilimab has shown similar efficacy compared with pembrolizumab. Compared to chemotherapy combined with sintilimab, there was no significant difference in progression-free survival (PFS, 8.9 months:9.0 months), objective response rate (ORR, 51.9\%:48\%), and disease control rate (DCR, 86.8\%:84.6\%) (8), whereas sintilimab had higher safety and obvious economic advantages (9). In this case, we chose sintilimab combined with platinum-based chemotherapy. After 1 course of treatment, the tumor mass shrank by more than $30 \%$, collateral circulation formed, most of the symptoms disappeared, and clinical relief was achieved. After 3 courses of treatment, the tumor mass had shrunk by more than $90 \%$. Taking into account the toxicity of chemotherapy, we chose sintilimab monotherapy after the fourth course of treatment. The patient was treated with sintilimab every 3 weeks and has been maintained until now. At the last follow-up (2021-12-07), we observed that the patient was in a good state of mind and his neck symptoms did not recur. The patient was satisfied with the treatment he received.

Along with sintilimab maintenance treatment, the patient's condition is stable, and no new lesions and metastases were seen. Compared with stent implantation and chemotherapy, stent implantation may quickly relieve the symptoms of patients, but has a high recurrence rate after surgery. Due to stent displacement and thrombosis, 26.9\% of patients were shown to require secondary surgery (10). Chemotherapy is non-invasive and relieves the symptoms of more than $60 \%$ of patients (11). However, it can take more than 2 weeks for symptoms to be relieved and can lead to adverse reactions including fatigue, diarrhea, colitis, pneumonia, hypothyroidism, and acute kidney injury (AKI) of grade 3 and above (12). So far, in this case, only grade 2 adverse events (such as diarrhea and nausea) occurred, while no grade 3 or above adverse events occurred.

Compared with traditional chemotherapy directly killing tumor cells, the treatment focus of immunotherapy is more reflected in the patient's recovery of immunity to tumor cells, which means that the patient can continue to produce immune cells that can recognize tumors, and it has a good therapeutic effect on micrometastasis that cannot be recognized by images. Therefore, patients who use immunotherapy can produce continuous therapeutic effect and patients can have a better long-term prognosis. CtDNA, lncRNA and PD-1 have shown a certain effect in predicting the prognosis of patients with advanced lung cancer $(13,14)$, but further research is needed, and there is still a lack of accurate biomarkers to evaluate the efficacy.

In recent years, immunotherapy combined with chemotherapy has become the standard treatment for advanced lung cancer, though there has been no corresponding report on the treatment of advanced lung cancer accompanied by SVCS. In this case, the application of immunotherapy combined with chemotherapy relieved the symptoms of SVCS and provided a novel idea for treating patients with advanced lung cancer accompanied by SVCS. However, this case still had certain shortcomings. The long-term effectiveness of the patient's medication still needs to be verified in further clinical treatment. Additionally, due to the lack of means to predict patient risk, the patient should be followed up to monitor treatmentrelated serious adverse reactions that may occur in the subsequent treatment. With continuous research, in the future, lung cancer will be like a chronic disease. Although lung cancer cannot be cured, its impact on the survival of patients will be minimized.

\section{Acknowledgments}

Funding: This research was supported by the National Natural Science Foundation of China (No. 81960532) and the Guizhou Province Science and Technology Plan (No. Qiankehe Support [2021] General 073).

\section{Footnote}

Reporting Checklist: The authors have completed the CARE reporting checklist. Available at https://apm.amegroups. com/article/view/10.21037/apm-21-3674/rc

Conflicts of Interest: All authors have completed the ICMJE uniform disclosure form (available at https://apm. amegroups.com/article/view/10.21037/apm-21-3674/coif). The authors have no conflicts of interest to declare. 
Ethical Statement: The authors are accountable for all aspects of the work in ensuring that questions related to the accuracy or integrity of any part of the work are appropriately investigated and resolved. All procedures performed in this study were in accordance with the ethical standards of the institutional and/or national research committee(s) and with the Helsinki Declaration (as revised in 2013). Written informed consent was obtained from the patient for publication of this case report and accompanying images. A copy of the written consent is available for review by the editorial office of this journal.

Open Access Statement: This is an Open Access article distributed in accordance with the Creative Commons Attribution-NonCommercial-NoDerivs 4.0 International License (CC BY-NC-ND 4.0), which permits the noncommercial replication and distribution of the article with the strict proviso that no changes or edits are made and the original work is properly cited (including links to both the formal publication through the relevant DOI and the license). See: https://creativecommons.org/licenses/by-nc-nd/4.0/.

\section{References}

1. Siegel RL, Miller KD, Fuchs HE, et al. Cancer Statistics, 2021. CA Cancer J Clin 2021;71:7-33.

2. Straka C, Ying J, Kong FM, et al. Review of evolving etiologies, implications and treatment strategies for the superior vena cava syndrome. Springerplus 2016;5:229.

3. Bierdrager E, Lampmann LE, Lohle PN, et al. Endovascular stenting in neoplastic superior vena cava syndrome prior to chemotherapy or radiotherapy. Neth J Med 2005;63:20-3.

4. Tanaka K, Morita S, Ando M, et al. A randomized phase 3 study of maintenance therapy with S- 1 plus best supportive care versus best supportive care after induction therapy with carboplatin plus S-1 for advanced or relapsed squamous cell carcinoma of the lung (WJOG7512L).

Cite this article as: Guo L, Qu W, Wei Y, Liang L, Tang Y, Chen C, Ke X. Sintilimab plus chemotherapy achieved symptom relief in stage IV lung squamous cell carcinoma with superior vena cava syndrome: a case report. Ann Palliat Med 2022;11(1):401-406. doi: 10.21037/apm-21-3674
Cancer 2020;126:3648-56.

5. Mose S, Stabik C, Eberlein K, et al. Retrospective analysis of the superior vena cava syndrome in irradiated cancer patients. Anticancer Res 2006;26:4933-6.

6. Azizi AH, Shafi I, Shah N, et al. Superior Vena Cava Syndrome. JACC Cardiovasc Interv 2020;13:2896-910.

7. Karakhanian WK, Karakhanian WZ, Belczak SQ. Superior vena cava syndrome: endovascular management. J Vasc Bras 2019;18:e20180062.

8. Gadgeel S, Rodríguez-Abreu D, Speranza G, et al. Updated Analysis From KEYNOTE-189: Pembrolizumab or Placebo Plus Pemetrexed and Platinum for Previously Untreated Metastatic Nonsquamous Non-Small-Cell Lung Cancer. J Clin Oncol 2020;38:1505-17.

9. Zhang L, Mai W, Jiang W, et al. Sintilimab: A Promising Anti-Tumor PD-1 Antibody. Front Oncol 2020;10:594558.

10. Friedman T, Quencer KB, Kishore SA, et al. Malignant Venous Obstruction: Superior Vena Cava Syndrome and Beyond. Semin Intervent Radiol 2017;34:398-408.

11. Büstgens FA, Loose R, Ficker JH, et al. Stent Implantation for Superior Vena Cava Syndrome of Malignant Cause. Rofo 2017;189:423-30.

12. Magee DE, Hird AE, Klaassen Z, et al. Adverse event profile for immunotherapy agents compared with chemotherapy in solid organ tumors: a systematic review and meta-analysis of randomized clinical trials. Ann Oncol 2020;31:50-60.

13. Yousefi M, Ghaffari P, Nosrati R, et al. Prognostic and therapeutic significance of circulating tumor cells in patients with lung cancer. Cell Oncol (Dordr) 2020;43:31-49.

14. Wilkerson MD, Yin X, Hoadley KA, et al. Lung squamous cell carcinoma mRNA expression subtypes are reproducible, clinically important, and correspond to normal cell types. Clin Cancer Res 2010;16:4864-75.

(English Language Editor: C. Betlazar-Maseh) 